\title{
Effect of channel block on the spiking activity of excitable membranes in a stochastic Hodgkin-Huxley model
}

\author{
G Schmid, I Goychuk and P Hänggi \\ Institut für Physik, Universität Augsburg, D-86135 Augsburg, Germany \\ E-mail: hanggi@physik.uni-augsburg.de
}

Received 5 February 2004

Accepted for publication 7 April 2004

Published 29 April 2004

Online at stacks.iop.org/PhysBio/1/61

DOI: $10.1088 / 1478-3967 / 1 / 2 / 002$

\begin{abstract}
The influence of intrinsic channel noise on the spontaneous spiking activity of poisoned excitable membrane patches is studied by use of a stochastic generalization of the Hodgkin-Huxley model. Internal noise stemming from the stochastic dynamics of individual ion channels is known to affect the collective properties of the whole ion channel cluster. For example, there exists an optimal size of the membrane patch for which the internal noise alone causes a regular spontaneous generation of action potentials. In addition to varying the size of ion channel clusters, living organisms may adapt the densities of ion channels in order to optimally regulate the spontaneous spiking activity. The influence of a channel block on the excitability of a membrane patch of a certain size is twofold: first, a variation of ion channel densities primarily yields a change of the conductance level; second, a down-regulation of working ion channels always increases the channel noise. While the former effect dominates in the case of sodium channel block resulting in a reduced spiking activity, the latter enhances the generation of spontaneous action potentials in the case of a tailored potassium channel blocking. Moreover, by blocking some portion of either potassium or sodium ion channels, it is possible to either increase or decrease the regularity of the spike train.
\end{abstract}

\section{Introduction}

Following the study of Hodgkin and Huxley [1], most of the models of axons have treated the generation and propagation of action potentials using deterministic differential equations. Since the work of Lecar and Nossal [2] it became clear, however, that not only the synaptic noise but also the randomness of the ion channel gating itself may cause threshold fluctuations in neurons [3]. Therefore, channel noise which stems from the stochastic nature of the ion channel dynamics must be taken into account [3]. It impacts such features as the threshold to spiking and the spiking rate itself [4-10], the anomalous noise-assisted enhancement of transduction of external signals, i.e. the phenomenon of stochastic resonance [11-14] and the efficiency for synchronization [15], to name but a few such phenomena. The origin of the channel noise [3] is basically due to fluctuations of the mean number of open ion channels around the corresponding mean values. Therefore, the strength of the channel noise is mainly determined by the number of ion channels participating in the generation of action potentials. Interestingly, there is an optimal patch size for which the spike production becomes more regular [11, 12]. The objective of this work is to investigate how the regularity of the spiking can possibly be controlled for a given membrane patch size. Toxins such as tetraethylammonium (TEA) and tetrodotoxin (TTX) allow to reduce the number of working potassium or sodium ion channels, respectively, for an extended period of time [16]. Moreover, the densities of ion channels can be adapted by the living cell also dynamically [17, 18] over an extended time span which is needed, e.g., to express the required ion channel proteins in the membrane [18]. The effect of blocking the ion channels entails several different tendencies at the same time. For sodium ion channels, the reduction in the density of channels results in an increase of the activation 
barrier towards excitation from the resting state and, therefore, in the reduction of neuronal activity. On the other hand, however, the corresponding channel noise component will also be increased due to reduction of the absolute number of ion channels in the membrane patch. As a consequence, the increased channel noise will help to overcome the activation barrier and to initiate spontaneous spikes. A reduction in the density of potassium channels will in contrast generally result in lowering of the activation barrier (an increase in the excitability—see, e.g., in [19]) and, simultaneously, an increase in the recovery time which should favour longer interspike time intervals. Moreover, the reduction in the total number of potassium ion channels will also increase the corresponding channel noise component which is expected to lead to an increased variability of the refractory period. Which of these various concurrent effects will dominate is not clear $a$ priori. This depends both on the ion channel densities and on the size of the studied membrane patch. It is the main objective of this paper to study this highly nontrivial, subtle issue in a stochastic model which extends the Hodgkin-Huxley model of the neuronal excitability.

\section{A stochastic Hodgkin-Huxley model}

According to the Hodgkin-Huxley model the dynamics of the membrane potential $V$, measured throughout in this work in $\mathrm{mV}$, is given by

$$
\begin{gathered}
C \frac{\mathrm{d}}{\mathrm{d} t} V+G_{\mathrm{K}}(n)\left(V-E_{\mathrm{K}}\right)+G_{\mathrm{Na}}(m, h)\left(V-E_{\mathrm{Na}}\right) \\
+G_{\mathrm{L}}\left(V-E_{\mathrm{L}}\right)=0 .
\end{gathered}
$$

In equation (1), $C=1 \mu \mathrm{F} \mathrm{cm}^{-2}$ is the capacity of the cell membrane. Furthermore, $E_{\mathrm{Na}}=50 \mathrm{mV}, E_{\mathrm{K}}=-77 \mathrm{mV}$ and $E_{\mathrm{L}}=-54.4 \mathrm{mV}$ are the reversal potentials for the sodium, potassium and leakage currents, respectively. While the leakage conductance is assumed to be constant, $G_{\mathrm{L}}=$ $0.3 \mathrm{mS} \mathrm{cm}{ }^{-2}$, the potassium and sodium conductances read:

$$
G_{\mathrm{K}}(n)=g_{\mathrm{K}}^{\max } x_{\mathrm{K}} n^{4}, \quad G_{\mathrm{Na}}(m, h)=g_{\mathrm{Na}}^{\max } x_{\mathrm{Na}} m^{3} h,
$$

where $g_{\mathrm{K}}^{\max }=36 \mathrm{mS} \mathrm{cm}^{-2}$ and $g_{\mathrm{Na}}^{\max }=120 \mathrm{mS} \mathrm{cm}^{-2}$ denote the maximal conductances (when all the channels are open). In equation (2) we introduce the factors $x_{\mathrm{K}}$ and $x_{\mathrm{Na}}$ which are the fractions of working, i.e. non-blocked ion channels, to the overall number of potassium, $N_{\mathrm{K}}$, or sodium, $N_{\mathrm{Na}}$, ion channels, respectively. These factors are confined to the unit interval. Experimentally, they can be controlled by adding cell toxins such as tetraethylammonium (TEA) and/or tetrodotoxin (TTX) which completely block and disable potassium or sodium ion channels, respectively [16].

While the gating variables $n, m$ and $h$ describe the mean ratios of the open gates of the working channels, the factors $n^{4}$ and $m^{3} h$ are the mean portions of the open ion channels within the membrane patch. This follows from the fact that the gating dynamics of each ion channel is assumed to be governed by four independent gates, each of which can switch between an open and a closed conformation. The voltage-dependent opening and closing rates $\alpha_{x}(V)$ and $\beta_{x}(V)(x=m, h, n)$, read $[11,12]$ :

$$
\begin{aligned}
\alpha_{m}(V) & =\frac{0.1(V+40)}{1-\exp [-(V+40) / 10]}, \\
\beta_{m}(V) & =4 \exp [-(V+65) / 18], \\
\alpha_{h}(V) & =0.07 \exp [-(V+65) / 20], \\
\beta_{h}(V) & =\{1+\exp [-(V+35) / 10]\}^{-1}, \\
\alpha_{n}(V) & =\frac{0.01(V+55)}{1-\exp [-(V+55) / 10]}, \\
\beta_{n}(V) & =0.125 \exp [-(V+65) / 80] .
\end{aligned}
$$

Fox and Lu [8] have extended the Hodgkin-Huxley model by taking into account the fluctuations of the numbers of open ion channels around the corresponding mean values. Within the corresponding stochastic description, the gating variables become stochastic quantities obeying the following Langevin equations:

$\frac{\mathrm{d}}{\mathrm{d} t} x=\alpha_{x}(V)(1-x)-\beta_{x}(V) x+\xi_{x}(t), \quad x=m, h, n$,

with independent Gaussian white noise sources $\xi_{x}(t)$ of the vanishing mean. For an excitable membrane patch with $N_{\mathrm{Na}}$ sodium and $N_{\mathrm{K}}$ potassium ion channels, the noise correlations assume the following form:

$$
\begin{aligned}
\left\langle\xi_{m}(t) \xi_{m}\left(t^{\prime}\right)\right\rangle & =\frac{2}{N_{\mathrm{Na}} x_{\mathrm{Na}}} \frac{\alpha_{m}(V) \beta_{m}(V)}{\left[\alpha_{m}(V)+\beta_{m}(V)\right]} \delta\left(t-t^{\prime}\right), \\
\left\langle\xi_{h}(t) \xi_{h}\left(t^{\prime}\right)\right\rangle & =\frac{2}{N_{\mathrm{Na}} x_{\mathrm{Na}}} \frac{\alpha_{h}(V) \beta_{h}(V)}{\left[\alpha_{h}(V)+\beta_{h}(V)\right]} \delta\left(t-t^{\prime}\right), \\
\left\langle\xi_{n}(t) \xi_{n}\left(t^{\prime}\right)\right\rangle & =\frac{2}{N_{\mathrm{K}} x_{\mathrm{K}}} \frac{\alpha_{n}(V) \beta_{n}(V)}{\left[\alpha_{n}(V)+\beta_{n}(V)\right]} \delta\left(t-t^{\prime}\right) .
\end{aligned}
$$

The overall numbers of involved potassium and sodium ion channels are re-scaled by $x_{\mathrm{Na}}$ and $x_{\mathrm{K}}$, respectively, in order to disregard the blocked channels which do not contribute to the channel noise. With an assumption of homogeneous ion channel densities, $\rho_{\mathrm{Na}}=60 \mu \mathrm{m}^{-2}$ and $\rho_{\mathrm{K}}=18 \mu \mathrm{m}^{-2}$, the ion channel numbers are given by $N_{\mathrm{Na}}=\rho_{\mathrm{Na}} S, N_{\mathrm{K}}=\rho_{\mathrm{K}} S$, where $S$ is the size of the membrane patch. The number of working ion channels, i.e. the size of the excitable membrane patch $S$, respectively, determines the strength of the fluctuations and thus the channel noise level. With decreasing patch size, i.e. decreasing number of ion channels, the noise level caused by fluctuations of the number of open ion channels increases, cf equation $(5 a)-(5 c)$. It is worth noting that the ItôStratonovich dilemma of the interpretation of the studied system of stochastic differential equations does not appear since each of the noise sources $\xi_{x}(t)$ does not depend explicitly on the state of the corresponding variable $x[20,21]$.

\section{Poisoning in the deterministic Hodgkin-Huxley model}

Before discussing the impact of channel noise on the spontaneous spiking activity of the poisoned membrane patches, we first consider the role of channel toxins on the excitability and resting potential of the original HodgkinHuxley model which neglects the fluctuations of the number of open ion channels. Then, the equations for the gating dynamics [1] read: 

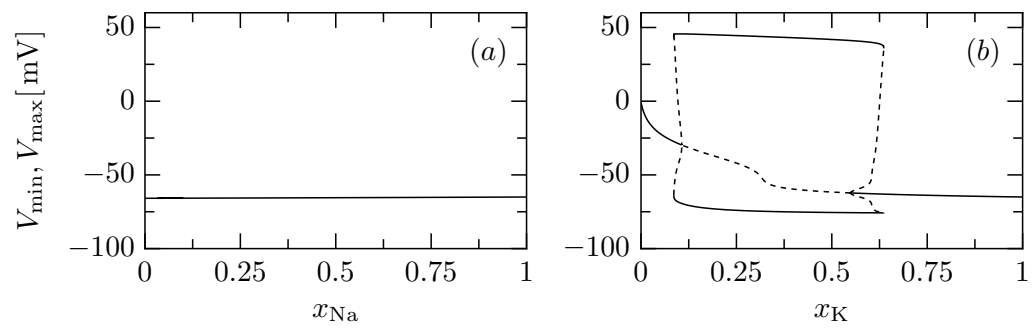

Figure 1. The bifurcation diagrams for the undriven original Hodgkin-Huxley model are plotted versus the ratio of intact sodium channels $x_{\mathrm{Na}}(a)$ and the ratio of intact potassium channels $x_{\mathrm{K}}(b)$, respectively, as bifurcation parameters. The solid line in $(a)$ denotes the stable fixed point solution. For potassium channel blocking $(b)$, the stable fixed point solution (single solid line) becomes unstable with decreasing $x_{K}$, and a stable spiking and oscillatory solution arise (the two solid lines refer to the maximal and minimal membrane potentials). The dashed lines correspond to unstable solutions.
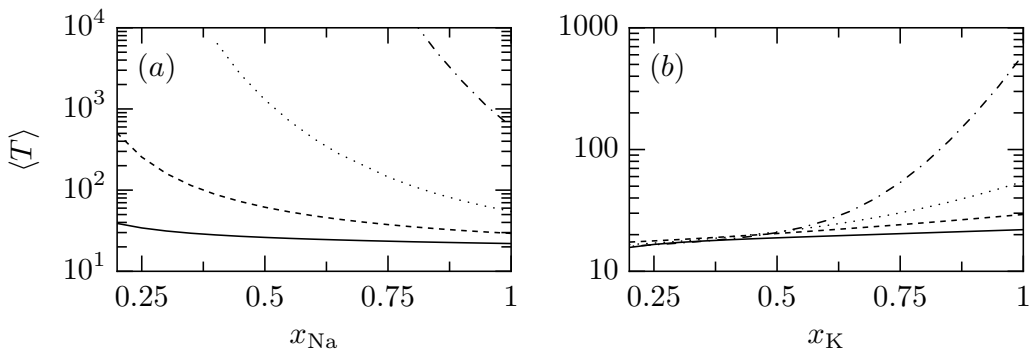

Figure 2. Mean interspike interval for poisoning: the dependence of the mean time between two subsequent, spontaneous action potentials versus $(a)$ the ratio of intact sodium channels and $(b)$ versus the fraction of active potassium ion channels is shown for four different patch sizes: $S=1 \mu \mathrm{m}^{2}$ (solid line), $S=4 \mu \mathrm{m}^{2}$ (dashed line), $S=16 \mu \mathrm{m}^{2}$ (dotted line) and $S=64 \mu \mathrm{m}^{2}$ (dashed-dotted line).

$$
\frac{\mathrm{d}}{\mathrm{d} t} x=\alpha_{x}(V)(1-x)-\beta_{x}(V) x, \quad x=m, h, n,
$$

with the opening and closing rates given by equations $(3 a)$ $(3 f)$. Hence equations (1), (2) and (6) form a deterministic Hodgkin-Huxley model which takes poisoning into account. Remarkably, this set of equations corresponds to the limit of infinitely large numbers of ion channels within the stochastic generalization which was introduced in section 2 .

Figure 1 depicts the bifurcation scenario which is derived from the deterministic model for the case of poisoning the membrane cluster. The poisoning of sodium channels causes only a small, practically negligible variation of the resting voltage, see figure 1 $(a)$. Obviously it cannot—upon neglecting the role of channel noise-induce spiking events. In contrast, a reduction in the number of working potassium channels changes dramatically the qualitative behaviour of the spiking activity, see figure 1(b). Upon decreasing the number of intact potassium channels, a sub-critical Hopf-bifurcation takes place - a stable spiking and oscillatory solution arises and the stable, non-spiking solution becomes unstable. Moreover, there is a region between $0.549 \leqslant x_{\mathrm{K}} \leqslant 0.636$, where a stable spiking and a stable fixed point solution coexist. With further reduction in the ratio $x_{\mathrm{K}}$, the oscillatory spiking solution loses stability, a sub-critical Hopf-bifurcation takes place and a stable fixed point solution arises again. Once more, a region of bistability within $0.0859 \leqslant x_{\mathrm{K}} \leqslant 0.1068$ is identified.

\section{The mean interspike interval}

The numerical integration of the stochastic generalized Hodgkin-Huxley model, cf equations $(1)-(5 c)$, is carried out by the standard stochastic Euler algorithm with a step size of $1 \mu \mathrm{s}$. The Gaussian random numbers are generated by the 'numerical recipes' routine ran2 using the BoxMuller algorithm [22]. To ensure the confinement of the gating variables between 0 (all gates are closed) and 1 (all gates are open), we have implemented numerically reflecting boundaries at 0 and 1 . The occurrences of action potentials are determined by upward crossings of the membrane potential $V$ of a certain detection threshold. Due to very steep increase in the membrane potential at firing the actual choice of the detection threshold does not affect the results. In our simulations the spontaneous spikes are found by upward crossings at zero threshold voltage. The occurrences of action spikes $t_{i}, i=1, \ldots, N$ form a point process.

Unlike the original, deterministic Hodgkin-Huxley model, where the action potentials occur only for certain superthreshold current stimuli, the intrinsic channel noise alone initiates spontaneous spikes [3-7, 9]. The mean interspike interval, i.e.

$$
\langle T\rangle=\frac{1}{N} \sum_{i=1}^{N}\left(t_{i}-t_{i-1}\right),
$$

with $t_{0}=0$, becomes a function of the patch size $S$. The inverse mean interspike interval defines the spiking rate. With the increasing noise level or decreasing patch sizes $S$, the spike production increases respectively, and thus the mean interspike interval $\langle T\rangle$ decreases and can approach the refractory time $[11,12]$.

In figure 2 we depict the mean interspike interval $\langle T\rangle$ against the fractions $x_{\mathrm{Na}}$ and $x_{\mathrm{K}}$ of the working sodium channels or potassium ion channels, respectively, for different 

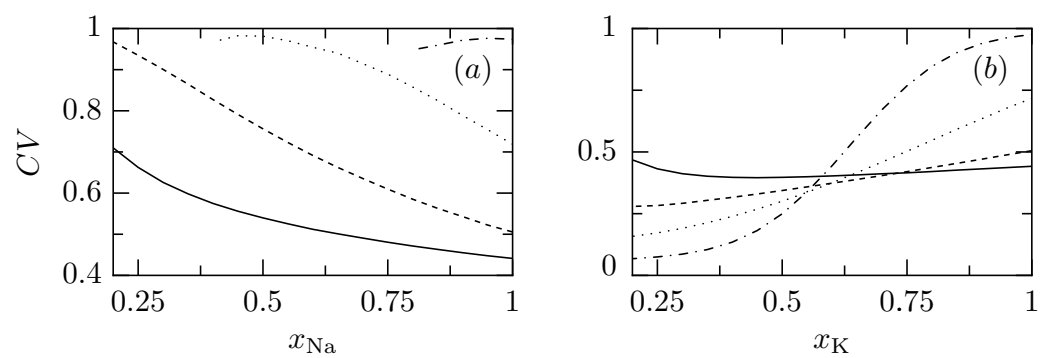

Figure 3. Same as in figure 2, but for the coefficient of variation $C V$, cf equation (8). By addition of TEA or TTX the regularity of the spike output can be both increased and decreased.

patch sizes $S$. A reduction in the number of working sodium ion channels, i.e. smaller $x_{\mathrm{Na}}$ values, increases the strength of channel noise which is caused by the stochastic behaviour of the sodium ion channels. Because the channel noise, which is induced by the sodium channels, is seemingly responsible for the initiation of action potentials from the rest potential [9]; one might expect that a reduction in the number of sodium ion channels could then lead to more spikes. Concurrently, however, a reduction in the number of working sodium channels causes a diminishment of the maximal sodium conductance. Given this competition between these two mechanisms, it is the latter effect that dominates and, consequently, causes an increase in the mean interspike interval, cf figure 2(a).

The reduction in potassium conductance by poisoning the potassium channels changes dramatically the dynamics of the original, deterministic Hodgkin-Huxley model which ignores the impact of the channel noise. With decreasing potassium conductance a stable spiking solution emerges at $x_{\mathrm{K}}=0.636$. The stable silent solution coexists with the stable spiking solution until $x_{\mathrm{K}}=0.549$. Depending on the initial value, the deterministic system spends the time either in the silent state, or on the limit cycle for $0.549<x_{\mathrm{K}}<0.636$. Consequently, the noisy system undergoes noise-induced stochastic temporal transitions between both stable manifolds. The motion on the spiking limit cycle becomes randomized. For $x_{\mathrm{K}}<0.549$, the stable fixed point disappears and the dynamics takes place on a stochastic limit cycle manifold. Then, the noise does not seem to affect strongly the mean period of the stochastic cycling. For different patch sizes this mean period $\langle T\rangle$ is about the same, cf figure $2(b)$.

However, before the Hopf-bifurcation occurs, the increase in the potassium channel noise can enhance the mean frequency of the stochastic spikes occurrences. Here, the mean interspike interval $\langle T\rangle$ can be drastically decreased with a reduction in working potassium channels $x_{\mathrm{K}}$, cf figure $2(b)$. This behaviour is rather different from that known before. It is generally appreciated $[3,9]$ that the fluctuational increase in the number of open sodium channels is the reason for the spontaneous spike generation. The role of the potassium channel noise is usually thought to be negligible. However, the decrease in the potassium conductance is known to enhance the excitability and it can even induce spikes deterministically (see before). Therefore, the fluctuational decrease in the number of open potassium channel can also induce spikes. Note in this respect that about one third of the potassium channels is open at the resting potential. This is contrary to the sodium channels which are almost all closed at the resting potential. The described alternative mechanism of the fluctuational spike generation can be clearly seen at work in figure $2(b)$ where the sodium channel noise remains unchanged for each plotted curve. The effect strongly depends, however, on the size of the membrane patch. For a sufficiently large membrane patch (dashed-dotted line in figure $2(b)$ ) the discussed effect is indeed very strong. However, when the patch size becomes small (solid line in figure $2(b)$ ), the poisoning of potassium channels does not cause much effect since the stochastic dynamics is dominated in this case by the channel noise component stemming from the stochastic dynamics of sodium channels. These qualitative features are displayed in figure $2(b)$.

\section{Controlling the coherence of poisoned spiking activity}

We next address the regularity of spontaneous action potentials. A proper measure is the coefficient of variation, $C V$, a measure of coherence, which is given as the ratio of standard deviation to the mean value

$$
C V=\frac{\sqrt{\left\langle T^{2}\right\rangle-\langle T\rangle^{2}}}{\langle T\rangle},
$$

where $\left\langle T^{2}\right\rangle:=\frac{1}{N} \sum\left(t_{i}-t_{i-1}\right)^{2}$ is the mean-squared interspike interval. For a fully disordered point process (the case of Poisson process) the coefficient of variation $C V$ assumes the value $C V=1$, while for more ordered processes it assumes smaller values and for a deterministic signal it vanishes. In previous studies it has been demonstrated that $C V$ exhibits a distinct minimum for an optimal patch size $S \approx 1 \mu \mathrm{m}^{2}$ at which the spiking is mostly regular $[11,12]$. This phenomenon has been termed an intrinsic coherence resonance.

In figure 3 , the coefficient of variation is plotted versus the fractions $x_{\mathrm{Na}}(a)$ and $x_{\mathrm{K}}(b)$. Any addition of TTX which blocks sodium ion channels leads to an increase in the $C V$, cf figure $3(a)$. In contrast, toxins which disable potassium channels yield a rise of the regularity, even beyond the level which can be reached for an optimal patch size with the unmodified density of the ion channels, cf [11]. This result is due to the fact that the lowering of the potassium conductance level promotes a stable oscillatory spiking solution in the 
deterministic model. The channel noise, in contrast, tends to disturb the regular spiking. Nevertheless, the former effect dominates resulting in the observed behaviour for the $C V$. In the case of optimal patch size, where the phenomenon of intrinsic coherence resonance occurs, the poisoning of potassium channels does not produce a strong effect. This is in contrast with the poisoning of sodium channels which destroys the coherence resonance (solid line in figure 3(a)).

\section{Conclusion and outlook}

Our study of the stochastically generalized Hodgkin-Huxley model reveals the possibility of manipulating the response of a spiking membrane patch by adding toxins which selectively block ion channels. For example, by a fine-tuned addition of tetrodotoxin (TTX) a certain portion of sodium ion channels could be experimentally disabled on purpose. This in turn results in a reduction in the spontaneous action potentials and causes a less regular production of spikes. On the other hand, the addition of tetraethylammonium (TEA) can be used in order to block potassium ion channels. This causes a surprising increase in the spiking activity (i.e. a decrease in the mean interspike interval) and yields in turn a more regular spontaneous spiking coherence. These characteristic features are expected to impact as well the behaviour of biological 'stochastic resonance', i.e. the phenomenon that the application of an appropriate dose of noise can boost signal transduction $[13,14]$ and, also, the phenomenon of 'coherence resonance' [23] in oscillatory or excitable biological entities [11].

We share the confident belief that our study of the tailored control of channel noise via channel blocking in an archetypal model of excitable biological membranes provides some insight into the underlying principles and mechanisms and thus will motivate further studies of more realistic models of real neurons where such channel noise phenomena do play an essential and constructive role.

\section{Acknowledgment}

This work has been supported by the Deutsche Forschungsgemeinschaft via the Sonderforschungsbereich SFB-486, project A10.

\section{Glossary}

Action potential/spike. A rapid change of the transmembrane electrical potential in the excitable cell membrane.

Bifurcation. A qualitative change in the topology of the attractor-basin phase portrait under a small variation of a parameter within a nonlinear system.

Channel noise. The fluctuations of the number of open ion channels.

Coherence resonance. Noise-induced improvement in the regularity of the system's output.

Excitable membrane. Cell membranes that are capable of rapidly changing their trans-membrane electrical potential in the form of spikes.
Gates/gating. Closing and opening functions of an ion channel. This usually refers to the movement of protein structural elements of the channel that occludes the channel pore.

Gating variable. A variable which accounts for the fraction of open channel gates of a certain type.

Gaussian white noise. Stochastic trajectories whose distributions are uncorrelated in time and are Gaussian distributed.

Hopf-bifurcation. A bifurcation of a fixed point to an oscillatory solution. When the oscillatory solution is unstable and exists at subcritical values of the control parameter, we term it a subcritical Hopf-bifurcation. Conversely, when the oscillatory solution is stable and exists at supercritical values of the control parameter, a supercritical Hopf-bifurcation is observable.

Ion channel. A protein folded in the cell membrane which enables the transport of specific ions through the membrane.

Itô-Stratonovich dilemma. Interpretation problem which arises in the context of Langevin equations in case of multiplicative Gaussian white noise.

Langevin equation. An equation of motion that describes the temporal evolution of a variable which is subjected to noise acting on the system.

Limit cycle. An attracting set of a nonlinear system to which trajectories converge. Upon the limit cycle these trajectories are closed and periodic.

Manifold. A topological space wherein a stable solution of a nonlinear system remains.

Membrane patch. A portion of the cell membrane with a certain size.

Membrane potential. The trans-membrane electrical potential.

Opening/closing rate. Under the two-state assumption for the gate dynamics (open and closed), these give the probability per time unit for transitions between the two states of a single gate.

Poisson process. A stochastic process which is memory-less (Markovian) with exponentially distributed waiting times between two successive events.

Recovery time. The time needed for relaxation to the resting state after an excitation occurred. Under physiological conditions an initiation of an action potential within this time span is not possible.

Reversal potential. The electrical potential for which the trans-membrane flux of specific ions vanishes. 
Rest potential/rest state. The equilibrium position of the trans-membrane potential of a certain membrane patch.

Spontaneous spiking activity. The occurrence of action potentials which are not initiated by an externally applied stimulus.

Stochastic resonance. A anomalous, noise-assisted enhancement of transduction of weak (deterministic or stochastic) signals.

\section{References}

[1] Hodgkin A L and Huxley A F 1952 J. Physiol. (London) 117500

[2] Lecar H and Nossal R 1971 Biophys. J. 111048

[3] White J A, Rubinstein J T and Kay A R 2000 Trends Neurosci. 23131

[4] Skaugen E and Walløe L 1979 Acta Physiol. Scand. 107343

[5] Clay J R and DeFelice L J 1983 Biophys. J. 42151

[6] Strassberg A F and DeFelice L J 1993 Neural Comput. 5843

[7] DeFelice L J and Isaac A 1993 J. Stat. Phys. 70339
[8] Fox R F and Lu Y N 1994 Phys. Rev. E 493421

[9] Chow C C and White J A 1996 Biophys. J. 713013

[10] Schneidman E, Freedman B and Segev I 1998 Neural Comput. 101679

[11] Schmid G, Goychuk I and Hänggi P 2001 Europhys. Lett. 5622

[12] Jung P and Shuai J W 2001 Europhys. Lett. 5629

[13] Gammaitoni L, Hänggi P, Jung P and Marchesoni F 1998 Rev. Mod. Phys. 70223

[14] Hänggi P 2002 Chem. Phys. Chem. 3285

[15] Schmid G, Goychuk I and Hänggi P 2003 Physica A 325165

[16] Hille B 2001 Ionic Channels of Excitable Membranes 3rd edn (Sunderland, MA: Sinauer Associates)

[17] LeMasson G, Marder E and Abbott L F 1993 Science 2591915

[18] Stemmler M and Koch C 1999 Nature Neurosci. 2521

[19] Goldman M S, Golowasch J, Marder E and Abbott L F 2001 J. Neurosci. 215229

[20] Hänggi P and Thomas H 1982 Phys. Rep. 88207 see section 6.2

[21] Hänggi P 1980 Helv. Phys. Acta 53491

[22] Press W H, Teukolsky S A, Vetterling W T and Flannery B P 1992 Numerical Recipes in C 2nd edn (Cambridge: Cambridge University Press)

[23] Pikovsky A S and Kurths J 1997 Phys. Rev. Lett. 78775 